\title{
Trouble conceiving after a Caesarean section - let's cut to the chase
}

\author{
Sinéad Maria 0 Neill \\ Department of Epidemiology \& Public Health/ Department of Obstetrics \& \\ Gynaecology, UCC
}

"Intellectual growth should commence at birth and cease only at death" (Albert Einstein)

\section{Caesarean section rates climb to an all-time high}

With birth by Caesarean section now being considered a very safe and convenient procedure, rates have peaked to the highest levels ever recorded worldwide. Surprisingly, more than one in four of all babies born in Ireland and one in three in the United States are born by Caesarean section. South American countries such as Brazil, Paraguay and Ecuador report Caesarean section rates of greater than $40 \%$ and Beijing in China reached an astounding rate of $51 \%$ in 2010 . So why the increase? Are women becoming "too posh to push"? Some of the proposed driving forces behind this massive rise include perceived benefits to the infant including minimal trauma as the baby will not travel through the birth canal. In addition, there is a very real fear of malpractice litigation among doctors putting them under extra pressure to carry out more Caesarean deliveries. The increasing age of first-time mothers (currently 31.2 years in Ireland) brings with it the potential for extra complications including low birth weight, gestational diabetes and stillbirth, and therefore an increased risk of having a Caesarean birth. Higher socioeconomic status, greater levels of education, private health insurance and personal preference are also attributed with the rise in Caesarean section rates. Some women fear painful natural births and choose an operation because they believe it to be less risky. The convenience of planning the date and time of a baby's birth by elective Caesarean delivery is also a basis for increasing rates. Surprisingly, some hospitals are also motivated by the higher fees associated with operative delivery. "Health before wealth" does not come into play in these hospitals unfortunately, nor does the enormous strain put on health resources and funding as a result of these extra costs in the form of additional staff input, longer length of stay in hospital, increased rates of infection and intensive care admissions. According to a recent World Health Organization (WHO) report "Caesarean sections have reached epidemic proportions in many countries worldwide and Caesareans, even when uncomplicated, have been shown to cost twice as much as a natural vaginal delivery". 




Figure 1: Too posh to push?

Whatever the reasons behind this massive rise in Caesarean sections worldwide, Caesarean delivery carries with it benefits as well as risks. A Caesarean delivery can be a lifesaving operation especially in emergency situations such as cases of very premature babies, or during labour if the baby goes into distress, or in the case of a very large baby. In addition, the operation itself has become much safer due to improvements in obstetrical training and surgical techniques and skills. A major benefit for mothers is that there is no labour pain in the case of maternally requested or planned Caesareans, in contrast to vaginal deliveries for which the duration of labour is unknown and may take many hours. The operation is in addition more private than a vaginal delivery it could be argued, quicker and can be scheduled in advance for convenience.

However, Caesarean delivery also carries risks for mother and baby. Risks to the mother include: infection at the site of the wound, rupture of the abdominal wall, blood clots and adhesions and in rare cases, even death. Moreover, the long-term consequences of a Caesarean section are now a matter of concern, as there is some evidence that operative delivery is associated with subsequent sub-fertility (i.e. a longer time interval between pregnancies), ectopic pregnancy (a pregnancy which occurs outside of the womb, is fatal for the fetus and one of the leading causes of early pregnancy deaths in women), and spontaneous miscarriage, the most common complication of early pregnancy, occurring in $10-15 \%$ of recognised pregnancies. Robust, large scale, population level research is lacking to date, which is surprising considering the serious nature of these outcomes and the ever increasing trend of 'elective' Caesarean delivery.

\section{To have a Caesarean section or not to have a Caesarean section, that is the question}

This quantitative research seeks to clarify whether delivery by Caesarean section is associated with longer intervals between pregnancies, ectopic pregnancy and spontaneous 


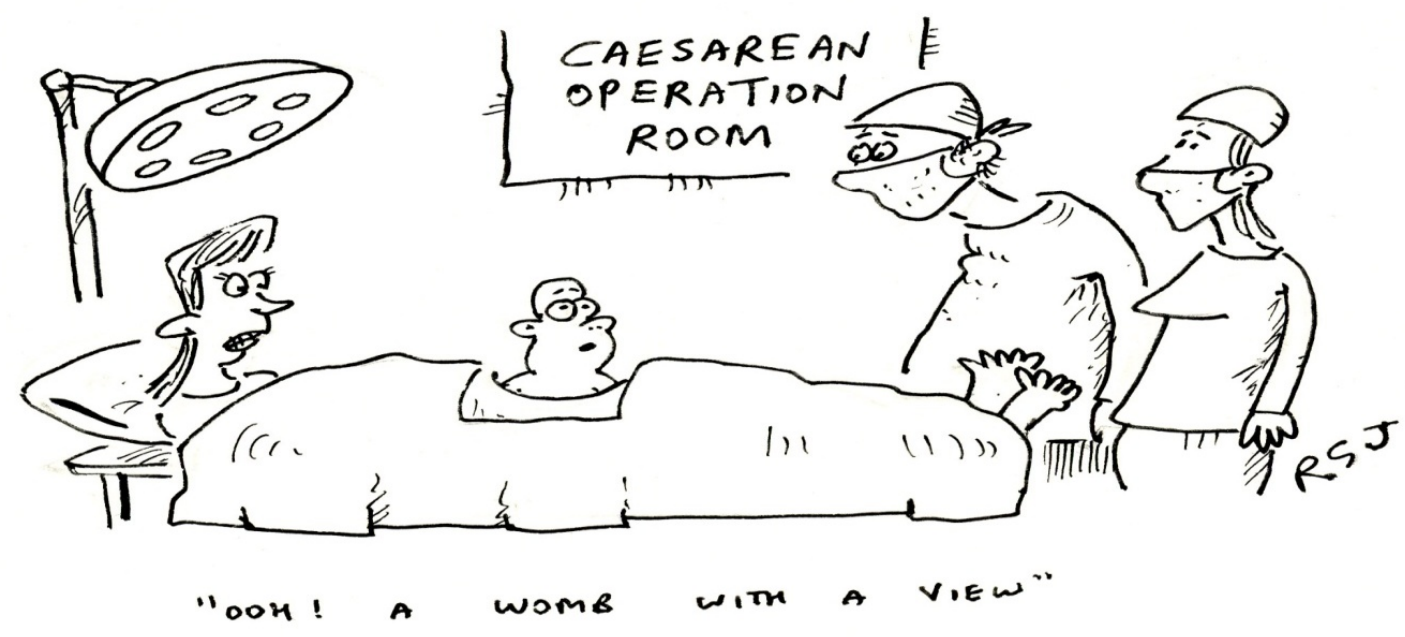

Figure 2: "Ooh! A womb with a view."

miscarriage by using a large Danish population-based dataset, with health information on women during and after pregnancy. I will travel to Aarhus in Denmark on various different trips to collaborate with the National Centre for Register-based Research (NCRR) staff. Here, I will be given access to this rich data source, the Civil Registration System (CRS) data, which is comprised of many different registers including the Medical Birth Register (MBR), the National Hospital Register (NHR) and the In-Vitro Fertilisation (IVF) Register to name but a few. The linkage of a mass amount of data from these various registries is possible due to the incorporation of a unique identifier, a personal identification number, which is a six digit code given to each person alive or living in Denmark and stays with them until death. Registration is compulsory by law and so coverage is over $99.9 \%$ complete. Few countries have such detailed data available and this study will be the largest study of its kind to date. The size of the population to be included (over 800,000 women) will add to the power of the study and improve the trustworthiness and reliability of the results. A statistical test known as survival analysis will be used to answer the research question by comparing women who had a previous Caesarean section with women who had a previous vaginal delivery and following them up to their next pregnancy. The rates of ectopic pregnancy, spontaneous miscarriage and time to next pregnancy will then be compared between the two groups to investigate whether or not prior Caesarean delivery is responsible.

This research will have potential impact nationally and internationally, regardless of whether a positive or negative association between Caesarean delivery and subsequent pregnancy loss is found. First and foremost, the findings will better inform women's decisions regarding delivery choice. We should never forget that Caesarean delivery is a major abdominal surgery and every surgery poses a risk. Education is the key to informed decision making and if women are made more aware of the potential benefits as well as risks associated with not only operative delivery, but all modes of delivery, then they will make the best 
choice that suits their individual situation in conjunction with their healthcare providers. Secondly, the findings may influence policy makers and their decisions regarding healthcare budgets and expenditure. Finally, in light of the recently published UK National Institute for Health and Clinical Excellence (NICE) guidelines, which support a woman's right to request a Caesarean delivery without medical reason, there is an urgent need to establish whether mode of delivery has a causal effect on risk of infant death and a woman's overall fertility. This research is imperative as Caesarean section rates are now at the highest ever recorded, and unfortunately fertility is becoming more of an issue with one in six couples having trouble conceiving. Moreover, with only a handful of studies existing to date with conflicting results, inadequate sample sizes and poor research methods, the question of which mode of delivery really is the safest becomes crucial. Ultimately, the aim of this research is to delve further into the often controversial mode of delivery and subsequent sub-fertility question and add to the field of research to date. In the case of delivery by Caesarean section, "the first cut may not be the deepest but it may have lifelong affects".

Thanks to my supervisory panel: Dr. Ali Khashan, Dr. Patricia Kearney, Professor Louise Kenny, Ms. Jennifer Lutomski and Professor Richard Greene, as well Professor Esben Agerbo and his colleagues at the National Centre for Registry-Based Research [NCRR] in Aarhus Denmark. Finally, thank you to my funding sources, the National Perinatal Epidemiology Centre of Ireland [NPEC] and the Irish Health Research Board [HRB].

Images from CartoonStock.com 